\title{
The Nijmegen Questionnaire and dysfunctional breathing
}

\author{
Jan van Dixhoorn ${ }^{1,2}$ and Hans Folgering ${ }^{3}$ \\ ${ }^{1}$ Centre for Breathing Therapy, Amersfoort, The Netherlands. ${ }^{2}$ Science Bureau, Linneaus Institure, Haarlem, \\ The Netherlands. ${ }^{3}$ Emeritus Professor of Respiratory Physiology, Nijmegen, The Netherlands.
}

Correspondence: Jan van Dixhoorn, Centre for Breathing Therapy, F. Van Blankenheymstraat, 10 Amersfoort, 3817 AG, The Netherlands. E-mail: vdixhoorndeuronet.nl

@ERSpublications

The Nijmegen Questionnaire is useful to quantify and assess the normality of subjective sensations http://ow.ly/MBJj1

The Nijmegen Questionnaire was introduced over 30 years ago as a screening tool to detect patients with hyperventilation complaints that could benefit from breathing regulation through capnographic feedback [1]. It was validated against the Hyperventilation Provocation Test [2], on the assumption at the time that hypocapnia was causally related to complaints. In later studies, the correlation between Nijmegen Questionnaire scores and carbon dioxide tensions appeared highly variable. The diagnosis of hyperventilation syndrome (HVS) was questioned and slowly disappeared. Nevertheless, the Nijmegen Questionnaire has remained in use and we have noticed even a recent increase in studies in clinical medicine as well as requests for permission to use the Nijmegen Questionnaire in new studies. We would like to formally declare here that the Nijmegen Questionnaire is not copyrighted and is free to use. It has been or will be translated, as far as we know, into Greek, Farsi, Finnish, Norwegian, Swedish, Spanish, Filipino and Chinese. However, the validity of the Nijmegen Questionnaire is often a point of discussion and we would like to make a few comments on that issue [3].

\section{Elevated Nijmegen Questionnaire score is not diagnostic of a specific syndrome}

We retrieved the datasets of our original validation studies and computed the average scores of HVS and non-HVS subjects [2, 4]. For the first validation study [2], 263 subjects were included. They were all patients from a psychiatric or internal medicine outpatient clinic or a lung function laboratory. Patients who experienced their main complaints after voluntary hyperventilation were compared to patients who did not. In the second validation study [4], a group of physician-diagnosed, nonasthmatic hyperventilators were compared to nonasthmatic normal controls. Figure 1 shows that the hyperventilators score equally highly on the Nijmegen Questionnaire in both studies but the controls were patients in the first study (Nijmegen Questionnaire score 19.5 \pm 10.5 ) and normal subjects in the second (Nijmegen Questionnaire score 11.9 \pm 5.5 ). The cut-off point for optimal differentiation appeared to be a sum score of $>22$ in the first study. In the second study, we calculated percentages to correct classification for different values of the sum score. We shuffled across possible cut-off points and found percentages correctly classified at $>17$ (90.6\%), $>18$ (94\%) and $>19$ (92.6\%). Thus, a score of $\geq 19$ best differentiates hyperventilatory patients from normal subjects. It shows that these complaints "are not part of the minor disturbances that commonly occur" and are not "part of normal life" [4].

Neither study attempted to differentiate "hyperventilation" complaints from anxiety disorder or other stress- or anxiety-related problems, nor has this been done in later studies. Thus, a high Nijmegen Questionnaire score does not indicate a specific syndrome. Both studies basically offer evidence that an elevated Nijmegen Questionnaire score is "abnormal". The Nijmegen Questionnaire is therefore a valid screening tool to detect patients across several medical specialties for whom the complaints may not be

Received: March 042015 | Accepted after revision: May 062015

Conflicts of interest: Disclosures can be found alongside the online version of this article at openres.ersjournals.com

Copyright CERS 2015. This article is open access and distributed under the terms of the Creative Commons Attribution Non-Commercial Licence 4.0. 


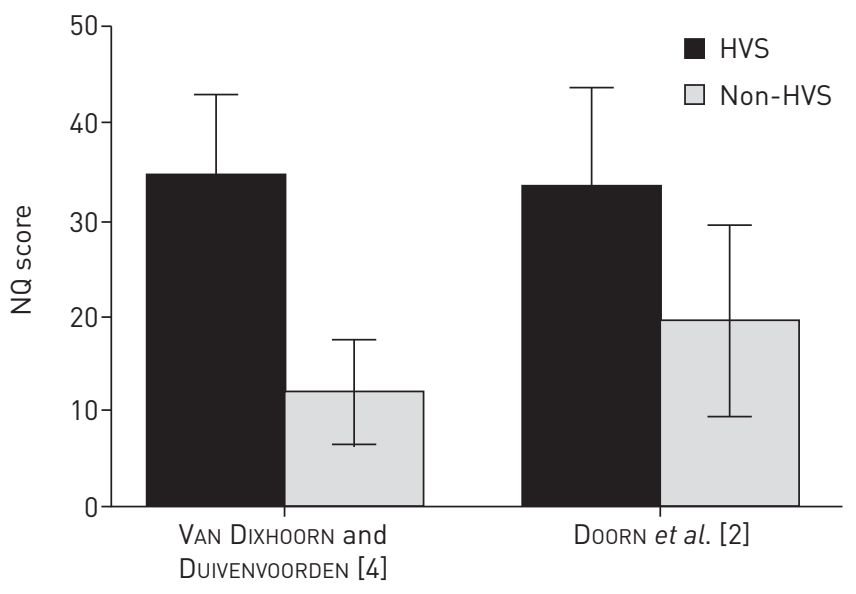

FIGURE 1 Nijmegen Questionnaire (NQ) scores (mean \pm sD) of hyperventilation syndrome (HVS) and non-HVS subjects in two validation studies.

(fully) due to physiological abnormality. It detects transdiagnostic and probably nonmedical abnormality. Most studies in clinical medicine, like pulmonology or otorhinolaryngology, do indeed use the Nijmegen Questionnaire to detect patients for whom something other than the medical condition may be a cause of the complaints and, thus, medical treatment should be reconsidered. For instance, Hanna et al. [5] used the Nijmegen Questionnaire as a screening test for nasal congestion in an otorhinolaryngology clinic and found 28 out of 118 patients to have elevated scores. In 12 of the 28 patients, the original diagnosis was reconsidered. They commented that the major benefit is "that a patient is spared an unnecessary and unhelpful procedure and the overall success of surgery is improved through better patient selection". This corresponds with a major motivation in the first report on HVS, that "early detection of these tension related complaints would prevent unnecessary visits to medical specialists and treatment" [2].

The validity and cut-off score of the Nijmegen Questionnaire to detect "abnormality" depends on a comparison with normal values in the same setting and culture in which the questionnaire is used. Normal values were found in several studies in English, Belgian and Dutch subjects [6-8] and range from 10-12 \pm 7 . By contrast, normal Chinese subjects score much lower: $4.7 \pm 4.6$ [7].

\section{The Nijmegen Questionnaire reflects a subjective aspect of dysfunctional breathing}

The nature of the abnormality is a subject for discussion. Inspection of the 16 items of the Nijmegen Questionnaire indicates that the sum score is related to stress, respiration and anxiety. They overlap with symptoms of panic disorder but only a subgroup of patients with high scores on the Nijmegen Questionnaire have been actually diagnosed as having panic disorder [7]. Many asthmatics have an elevated Nijmegen Questionnaire score [9, 10], which corresponds with poor asthma control [9, 11]. Breathing pattern disorder is also related to the Nijmegen Questionnaire score [12]. The relationship with hypocapnia is variable and often too weak to call the Nijmegen Questionnaire score a measure of hyperventilation [9, 13]. Thus, we prefer to describe the Nijmegen Questionnaire score a measure of "functional respiratory complaints". The word respiratory refers to ventilation, dyspnoea and breathing movement; the word functional refers to the relationship with stress and anxiety.

In the pioneering article by Thomas et al. [10], the term "dysfunctional breathing" was used instead of hyperventilation. This introduced the concept that breathing may be functionally disturbed beyond and even without hypocapnia. This wider concept of dysfunctional breathing is possibly the reason for the increasing popularity of studying it, but at the same time raises the question of its definition and measurement $[14,15]$. It is important to realise that breathing has several global functions, each of which can become dysfunctional $[13,16]$. Firstly, breathing has the function of air transport and diffusion (lung function). Secondly, the process of expansion and contraction of the trunk not only serves as the "air pump" but has also an important function in posture and movement (biomechanical function) [17]. Thirdly, the subjective experience of breathing is an important contributor to the "sense of self" (psychic function) $[18,19]$. All three functions can cause "dyspnoea" and are susceptible to stress. Thus, the lack of clarity over dysfunctional breathing is realistic when taking the multidimensionality of breathing into account. Multicomponent assessment is required. In the clinical evaluation of the presence of dysfunctional breathing, therefore, objective criteria, derived from the somatic functions of breathing, play an important role $[9,20]$. It seems desirable, therefore, to use at least two kinds of criteria, subjective and objective, to assess dysfunctional breathing. The Nijmegen Questionnaire mainly reflects the subjective, psychic dimension of breathing and its response to stress. 


\section{Normalisation of the Nijmegen Questionnaire score after breathing regulation}

One original intention of the Nijmegen Questionnaire was to have a tool to evaluate whether subjects with high scores would benefit from breathing regulation. There are many forms of breathing regulation and there is, at present, no evidence for any particular approach. However, it probably involves rather intense education and training to reverse the abnormal pattern and improve proper "use" of the body. For instance, Holloway and West [21] guided asthmatics, in five individual sessions, to re-educate them towards relaxed posture and breathing habits. Afterwards, Nijmegen Questionnaire values normalised completely to an average value of 12 . The implication of this is two-fold. First, on average, asthmatics do not have to have elevated Nijmegen Questionnaire scores. Asthma per se is not sufficient to increase the score. Secondly, when breathing regulation is able to solve the problem that caused the high Nijmegen Questionnaire score, the score does indeed normalise to around 12. In other studies of individual breathing regulation of patients without comorbidity, normalisation of Nijmegen Questionnaire values post-treatment was also found $[12,22]$. By contrast, simple breathing instructions from self-help manuals do not have any effect [23].

Evaluation of treatment by the Nijmegen Questionnaire is useful in clinical decisions at the individual level. Subjects whose scores remain elevated or in the upper-normal range after adequate breathing regulation treatment deserve further attention to seek out the reason for the lack of normalisation. There could be a hidden lung function disturbance, persistent anxious cognitions, persistent stressful life situation or any other reason, which needs to be addressed in another way than continuing breathing regulation. For instance, HAN et al. [24] studied 92 patients suspected with "hyperventilation complaints", without comorbidity. They received breathing regulation and, afterwards, showed a significant average decrease in Nijmegen Questionnaire score, but did not normalise. Stratified by subjective benefit, however, only one third reported strong improvement in their complaints and their Nijmegen Questionnaire scores normalised to 9.4. For the others, breathing regulation helped a little or not at all and their Nijmegen Questionnaire scores remained elevated. It is commendable, therefore, to evaluate treatment outcome not only by average reduction in the Nijmegen Questionnaire score, but to also stratify changes in the score according to improvement in the main complaint. In clinical studies that use the Nijmegen Questionnaire as a screening tool for dysfunctional breathing, this is only rarely done [25].

\section{Conclusion}

Given the multidimensionality of breathing, a single criterion for establishing dysfunctional breathing is not sufficient: multicomponent assessment is recommended. This includes ventilatory parameters such as carbon dioxide tension, breathing movement parameters and subjective variables. The Nijmegen Questionnaire is useful to quantify and assess the normality of subjective sensations.

\section{References}

1 Doorn PV, Folgering HTM, Colla P. Control of the end-tidal $\mathrm{PCO}_{2}$ in the hyperventilation syndrome: effects of biofeedback and breathing instructions compared. Bull Eur Physiopathol Respir 1982; 18: 829-836.

2 Doorn PV, Colla P, Folgering HTM. Een vragenlijst voor hyperventilatieklachten [A questionnaire for hyperventilation syndrome]. De Psycholoog 1983; 18: 573-577.

3 Goyal V, Sly PD. How children with asthma breathe: have we been overlooking a problem? Eur Respir J 2013; 41 : 1008-1009.

4 Van Dixhoorn J, Duivenvoorden HJ. Efficacy of Nijmegen Questionnaire in recognition of the hyperventilation syndrome. J Psychosom Res 1985; 29-2: 199-206.

5 Hanna BC, Woodman P, Adair R. Assessing the role of chronic hyperventilation in patients with nasal congestion: our experience in 118 patients. Clin Otolaryngol 2012; 37: 155-158.

6 Thomas M, McKinley RK, Freeman E, et al. The prevalence of dysfunctional breathing in adults in the community with and without asthma. Prim Care Respir J 2005; 14: 78-82.

7 Han JN, Stegen K, Schepers R, et al. Subjective symptoms and breathing pattern at rest and following hyperventilation in anxiety and somatoform disorders. J Psychosom Res 1998; 45: 519-532.

8 Van Dixhoorn J, Hoefman JD. Hyperventilatieklachten in de fysiotherapiepraktijk [Hyperventilation syndrome in physiotherapy]. Ned T Fysiotherapie 1985; 95-7/8: 167-171.

9 Grammatopoulou EP, Skordilis EK, Georgoudis G, et al. Hyperventilation in asthma: a validation study of the Nijmegen Questionnaire - NQ. J Asthma 2014; 51: 839-846.

10 Thomas M, McKinley RK, Freeman E, et al. Prevalence of dysfunctional breathing in patients treated for asthma in primary care: cross sectional survey. BMJ 2001; 322: 1098.

11 De Groot EP, Duiverman EJ, Brand PL. Dysfunctional breathing in children with asthma: a rare but relevant comorbidity. Eur Respir J 2013; 41: 1068-1073.

12 Courtney R, Van Dixhoorn JJ, Anthonissen E, et al. Medically unexplained dyspnea: partly moderated by dysfunctional (thoracic dominant) breathing pattern. J Asthma 2011; 48: 259-265.

13 Courtney R, Greenwood KM, Cohen M. Relationships between measures of dysfunctional breathing in a population with concerns about their breathing. J Bodyw Mov Ther 2011; 15: 24-34.

14 Morgan MDL. Dysfunctional breathing in asthma: is it common, identifiable and correctable? Thorax 2002; 57: Suppl. 2, I31-II5.

15 Keeley D, Osman L. Dysfunctional breathing and asthma. BMJ 2001; 322: 1075-1076. 
16 Van Dixhoorn J. Hyperventilation and dysfunctional breathing. Biol Psychol 1997; 46: 90-91.

17 Barker N, Everard ML. Getting to grips with 'dysfunctional breathing'. Paediatr Respir Rev 2015; 16: 53-61.

18 Rosenkranz MA, Davidson RJ. Affective neural circuitry and mind-body influences in asthma. Neuroimage 2009; 47: 972-980.

19 Leupoldt AV, Sommer T, Kegat S, et al. The unpleasantness of perceived dyspnea is processed in the anterior insula and amygdala. Am J Crit Care Med 2008; 177: 1026-1032.

20 Hagman C, Janson C, Emtner M. Breathing retraining - a five-year follow-up of patients with dysfunctional breathing. Respir Med 2011; 105: 1153-1159.

21 Holloway EA, West RJ. Integrated breathing and relaxation training (the Papworth method) for adults with asthma in primary care: a randomised controlled trial. Thorax 2007; 62: 1039-1042.

22 Burken PV. Het hyperventilatiesyndroom: een vergelijkend onderzoek van twee behandelstrategieen [Hyperventilation syndrome: a comparative study of two treatment strategies]. Ned T Fysiotherapie 1996; 94-104.

23 Conrad A, Muller A, Doberenz S, et al. Psychophysiological effects of breathing instructions for stress management. Appl Psychophysiol Biofeedback 2007; 32: 89-98.

24 Han JN, Stegen K, DeValck C, et al. Influence of breathing therapy on complaints, anxiety and breathing pattern in patients with hyperventilation syndrome and anxiety disorders. J Psychosom Res 1996; 41: 481-493.

25 Bartley J. Nasal congestion and hyperventilation syndrome. Am J Rhinol 2005; 19: 607-611. 